\title{
SANDZ2000-1192C
}

\section{Current Status of Three-Dimensional Silicon Photonic Crystals Operating at Infrared Wavelengths (invited)}

\author{
Shawn-Yu Lin and J.G. Fleming \\ Sandia National Laboratories, P.O. Box 5800, Albuquerque, NM 87185, USA \\ M.M. Sigalas, R. Biswas and K.M. Ho \\ Ames Laboratory, Department of Physics and Astronomy, Iowa State University, Ames,

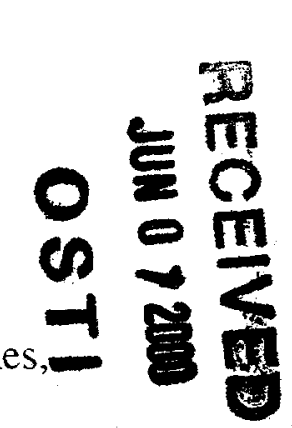
IA

\begin{abstract}
In this paper, the experimental realization and promises of three-dimensional (3D) photonic crystals in the infrared and optical wavelengths will be described. Emphasis will be placed on the development of new 3D photonic crystals, the micro- and nano-fabrication techniques, the construction of high-Q micro-cavities and the creation of 3D wave-guides.
\end{abstract}

\section{SAMPLE FABRICATION}

Our 3D silicon photonic crystals are fabricated using advanced silicon processing. The structure consists of layers one-dimensional rods, stacking according to certain crystal symmetry to form a lattice structure. A scanning-electron-microscopy image of the fabricated simple cubic structure is shown in Fig. 1 (a). The rod has a width of $w=0.8 \mu \mathrm{m}$ and the rod-to-rod spacing is $\mathrm{a}=3.2 \mu \mathrm{m}$. The dielectric filling fraction of the SC structure is $19 \%$. As the first step of the fabrication process, the square frame of width $w$ is lithographically patterned and etched. At the second step, a silicon dioxide layer is deposited to cover the patterned structure. The third step of the process is to use chemical-mechanical-polishing (CMP) to planarize the silicon dioxide surface. The fourth step is to deposite poly-silicon and then pattern it into the square posts, also with a width of $0.8 \mathrm{~mm}$ and a height of $\mathrm{h}=2.4 \mathrm{~mm}$. As the final step, silicon dioxide is deposited and then planarized using CMP. This completes the process for making a one-unit cell. The process may be continued to build more unit cells. In Fig.1(b), the transmission data taken from lattices are shown, respectively. A clear photonic band gap exists for SC lattices at $\lambda \sim 6 \mu \mathrm{m}$. 


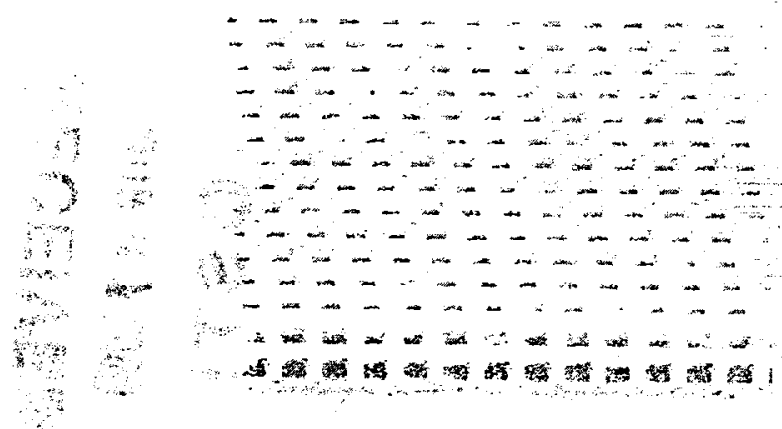

Fig.1 (a)

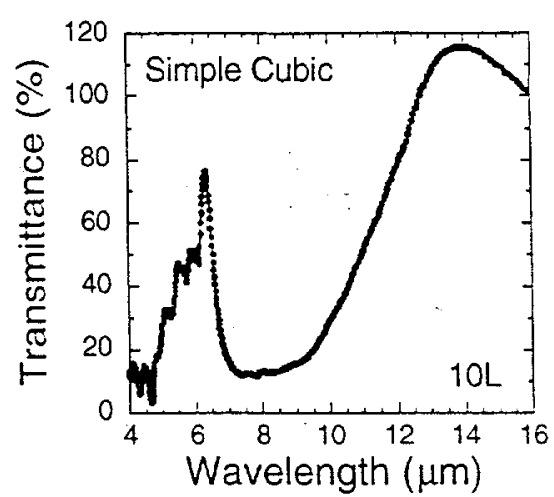

Fig. 1(b)

This micro-fabrication technique is powerful in that many different photonic crystal structures may be fabricated on the same six-inch silicon wafer. For example, a diamond-like lattice structure may be built together with a square lattice structure. In Fig.2(a), the SEM image of an eight-layer diamond lattice is shown. The transmission spectrum of the same structure but with ten-layers is shown in Fig.2 (b). Not only the spectral width of the photonic band gap is large, ranging from $\lambda=5.3 \mu \mathrm{m}$ to $7.7 \mu \mathrm{m}$, but also the attenuation is strong. $\mathrm{T}=2 \times 10^{-4}$, or equivalently a $37 \mathrm{~dB}$ attenuation at $\lambda=5.7 \mu \mathrm{m}$. The combination of a large photonic band gap and a strong attenuation make this diamond lattice structure well suited for many applications, ranging from signal guiding, routing, switching to spontaneous emission modification. It is believed that, by using this design, a ten to twelve layers structure is sufficient for most applications mentioned above.

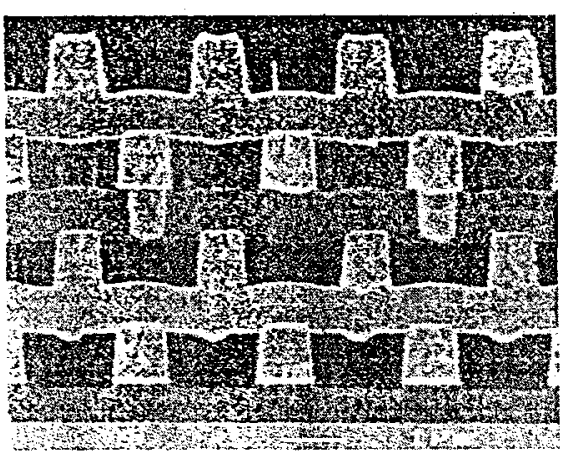

Fig. 2(a)

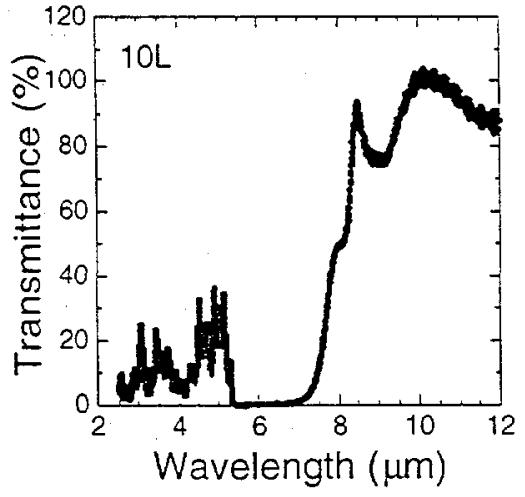

Fig.2(b) 


\section{DISCLAIMER}

This report was prepared as an account of work sponsored by an agency of the United States Government. Neither the United States Government nor any agency thereof, nor any of their employees, make any warranty, express or implied, or assumes any legal liability or responsibility for the accuracy, completeness, or usefulness of any information, apparatus, product, or process disclosed, or represents that its use would not infringe privately owned rights. Reference herein to any specific commercial product, process, or service by trade name, trademark, manufacturer, or otherwise does not necessarily constitute or imply its endorsement, recommendation, or favoring by the United States Government or any agency thereof. The views and opinions of authors expressed herein do not necessarily state or reflect those of the United States Government or any agency thereof. 


\section{DISCLAIMER}

Portions of this document may be illegible in electronic image products. Images are produced from the best available original document. 
In Fig.4(a) and (b), the transmission spectra from yet another type of 3D crystals, the HCP symmetry and the FCC symmetry, are shown. Using our technology, different types of crystal may be fabricated in the same six-inch wafer. Another example is the fabrication of $3 \mathrm{D}$ crystal using construction scheme other than the 1D rods line construction. The SEM images of two new designs are shown in Fig.5 (a) and (b), respectively. This type of design may be more suited for creating micro-cavities, as it is most natural to remove a single basic element while preserving the underlining crystal symmetry.

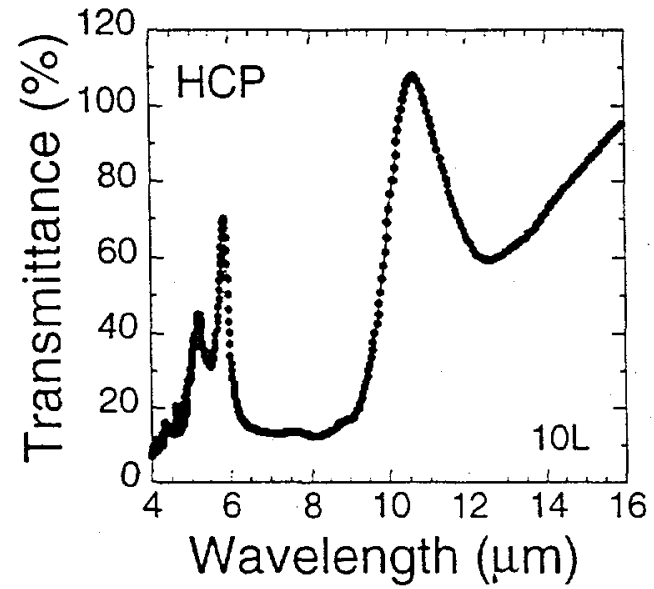

Fig.4(a)

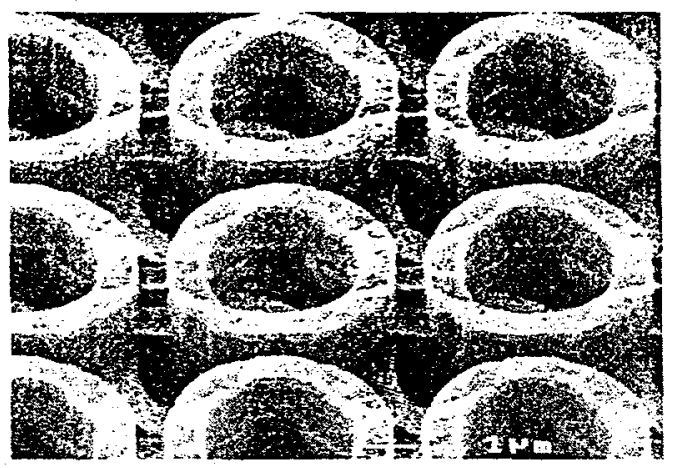

Fig.5(a)

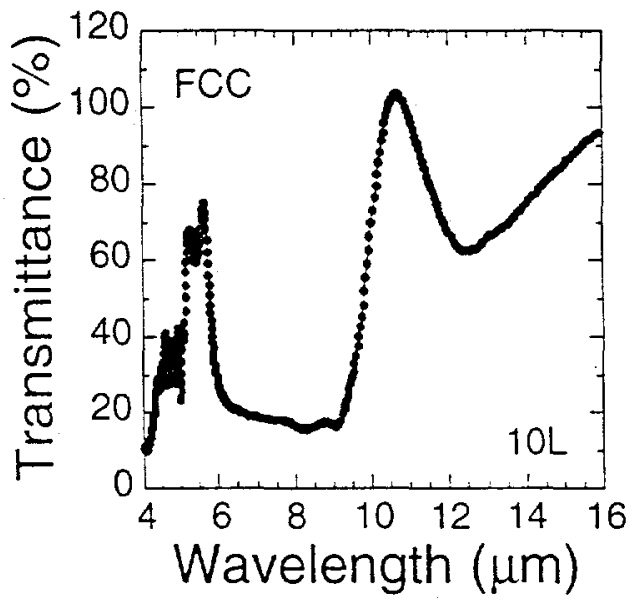

Fig.4(b)

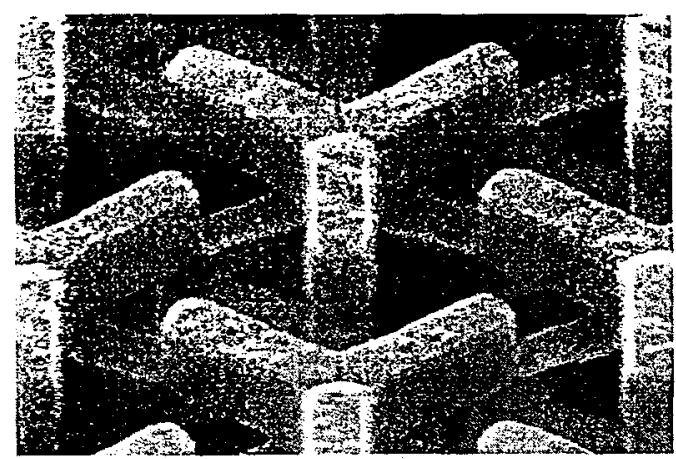

Fig. 5(b)

\section{D PHOTONIC CRYSTAL MICRO-CAVITIES}

A 3D micro cavity may be created by introducing defects to trap light at a point within a 3D photonic crystal. A defect is formed by changing the dielectric constant in a local region of the 
crystal. In particular, there are two classes of defects, namely the "vacancy defect" created by removing a section of the rod and an "interstitual defect" formed by adding extra dielectric materials into the crystal. The defect state may appears in the forbidden photonic band gap spectral regime, leading a strongly localized state. The strength of photonic localization is determined by the size of the band gap, which acts as a highly reflecting mirror. Previously, one type of vacancy defect was shown to have ultra small modal volume, $<\lambda^{3}$, and yet the cavity-Q value is small, $\sim 10$. A theoretical calculation suggests that an interstitual defect may give rise to a much higher cavity-Q value. A micro-cavity sample that is made of an eigth-layer diamond structure was successfully fabricated. The cavity consists of a small added section, $0.5 \mathrm{a}$, in the fifth layer of the structure. In Fig.3, a transmission spectrum of the micro-cavity is shown as a function of wavelength from 0 to $15 \mu \mathrm{m}$. At $\lambda=6.6 \mu \mathrm{m}$, there is clearly a sharp transmission peak, signifying the existence of a 3D cavity mode. Moreover, the cavity mode has a sharp linewidth of $\sim 20 \mathrm{~nm}$, shown in the inset of Fig.3, corresponding a cavity-Q of -300 . This is the highest cavity-Q ever achieved in any $3 \mathrm{D}$ photonic crystals in the infrared wavelengths.

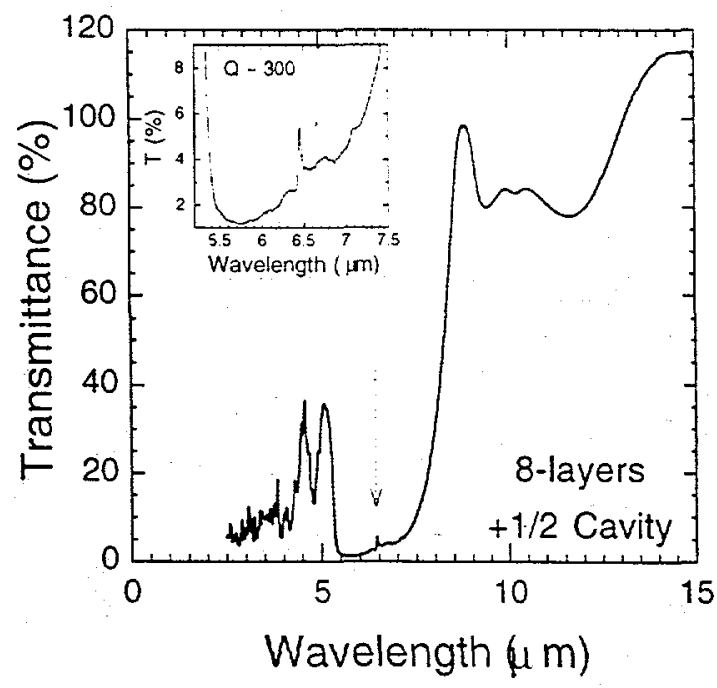

Fig. 3

3D WAVEGUIDES AND WAVEGUIDE BENDS 
Guiding of light around a sharp corner with high efficiency is important for large scale all-optical circuit and optical computing applications. Early theoretical simulation suggests that lossless guiding and bending of electromagnetic waves is possible using a $2 \mathrm{D}$ photonic crystal. Subsequently, a successful experimental demonstration was performed at mm-wave spectral range. Here, simulation on the full guiding and bending of light using 3D photonic crystals are a carried out.

A calculation of a 3D waveguide and waveguide bend in the diamond lattice structure is shown in Fig.6(a) and (b), respectively. The straight waveguide is created by removing one rod in the nth layer of the photonic crystal structure. The flux of electromagnetic wave is plotted as a function of frequency for a periodic structure (the black dashed line as a reference) and a straight waveguide (the blue line), respectively. The guiding mode covers about $50 \%$ of the photonic band gap. The waveguide bend is formed by removing one rod each from adjacent layers, i.e. the $\mathrm{N}^{\text {th }}$ and $\mathrm{N}+1^{\text {th }}$ in a diamond lattice. The joint of the two rods constitutes a 90-degree bend. The model profile in Fig.t(b) shows that a 100\% bending efficiency is attainable using such a bending configuration. The red color represents the photonic crystal and the green color the electric field.

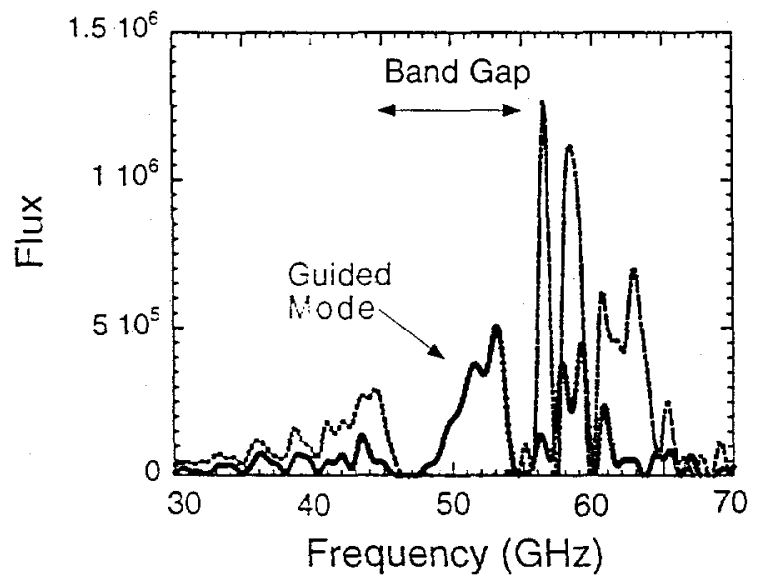

Fig.6 (a)

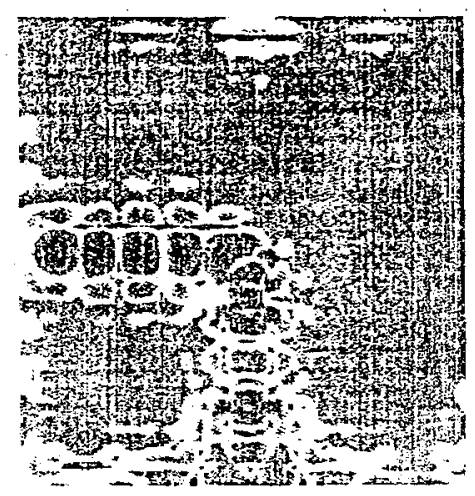

Fig. 6 (b) 


\section{SUMMARY}

In summary, an overview is given on the current status of three-dimensional (3D) photonic crystals. The realization of new 3D photonic crystal structures, the creation of high $Q$ microcavities and the building of waveguide bends are presented. These devices form the basic building blocks for applications in signal processing and low threshold lasers.

The work at Sandia National Laboratories is supported through DOE under contract no. DE-ACO494AL85000. Sandia is a multiprogram laboratory operated by Sandia Corporation, a Lockheed Martin Company, for the United States Department of Energy. Ames Laboratory is operated for the U.S. Department of Energy by Iowa State University under contract no. W-7405-Eng-82.

\section{REFERENCES}

1.S.Y. Lin et al., Nature, Vol. 394, 251-253 (1998).

2. .Fleming J.G. \& Lin S.Y, Opt. Lett., vol 24, 49 (1999).

3. S. Y. Lin et al., Phys. Rev. B R15; Vol.59, p.579-582 (1999).

4. S. Y. Lin et al., Science 282, 274-277 (1998).

5. M.M. Sigalas, R. Biswas, K.M. Ho, C.M. Soukoulis, D. Turner, B. Vasiliu, S.C. Kothari and S.Y. Lin, Microwave and optical technology letters 23, 56-59 (1999). 